\title{
La ablación intraósea por radiofrecuencia del nervio basicervical vertebral. ¿Solución en dolor discogénico refractario?
}

\author{
Radiofrequency intraosseous ablation of the vertebral \\ basicervical nerve. Solution in refractory discogenic pain?
}

\author{
J. Vidal Fuentes
}

Unidad del Dolor Reumático, Hospital Universitario de Guadalajara. Profesor clínico UAH. Madrid, España

El dolor lumbar crónico es unas de las patologías más prevalentes en la población general; supone un $10 \%$ de los procesos en países occidentes y la principal causa de discapacidad dentro de todos los procesos musculoesqueléticos (1). En España, según la Encuesta Nacional de Salud de 2017, el 20 \% de la población sufre lumbalgia crónica, prevalencia que aumenta con la edad [2]. El $95 \%$ de las causas de lumbalgia son de tipo mecánico, y el 26-39 \% son de origen discogénico [3].

Un estudio reciente de Fischgrund y cols. comunica los resultados a largo plazo de 5 años de un ensayo clínico sobre la efectividad de la ablación intraósea por radiofrecuencia del nervio basicervical vertebral en lumbalgia crónica [4]. La técnica bautizada como INTRACEPT ${ }^{\circledR}$ (https: //www. youtube.com/watch?v=Ht8kspBRZII) ha sido aprobada por la FDA en 2016.

El estudio en cuestión analiza la persistencia de eficacia tras un mínimo de seguimiento de 5 años en 100 pacientes de los 137 que recibieron dicho tratamiento activo del EC incial [5] (estudio en el que había grupo control simulado y que permitía el paso al grupo activo, de tal forma que el $75 \%$ de los pacientes recibieron dicho tratamiento]. Los criterios de selección eran: lumbalgia crónica sin respuesta a tratamiento conservador durante 6 meses, cambios radiológicos Modic 102 , limitación funcional en cuestionario Oswestry [ODI] severa y dolor $\geq 4 / 10 \mathrm{~cm}$. Se excluyeron la presencia de dolor radicular, cirugía previa, presencia de osteoporosis, protrusiones o hernias discales > de $5 \mathrm{~mm}$, espondilolistesis $>$ de $2 \mathrm{~mm}$, litigio o depresión. La técnica se realizó entre L3 y S1, con una duración entre 30 y 90’. La media de reducción del ODI fue del $60 \%$ y del dolor de 4,4 puntos. El $66 \%$ de los pacientes tuvo una reducción del dolor superior al $50 \%$, el $47 \%$ > del $75 \%$ y el $34 \%$ completa. Al menos un $75 \%$ de los pacientes tuvieron una mejoría clínica importante (reducción en 15 puntos del ODI y del dolor de 2 puntos, aceptadas cono diferencias mínimas clínicamente importantes]. Esta a mejoría se mantuvo en todos los pacientes a lo largo de los 5 años de seguimiento mínimo.

Los resultados de la cirugía como alternativa al tratamiento de la patología degenerativa discal, como el reemplazamiento discal o las técnicas de fusión vertebral, presentan unos resultados de mejoría clínica en dolor o en el ODI muy inferiores a las que ofrece el INTRACEPT ${ }^{\circledR}$, con un costes mayores, más días de hospitalización y complicaciones y peores resultados a largo plazo $(6,7)$.

El dolor discogénico es un reto terapéutico con limitaciones en el abordaje farmacológico, tanto por la eficacia como por la seguridad, sobre todo largo plazo, tanto de los AINE, los opioides o del propio paracetamol [8]. Las técnicas mínimamente invasivas sobre el disco, como las diferentes formas de radiofrecuencia, tienen escasa evidencia o no son suficientemente eficaces [9].

La posibilidad de identificar claramente pacientes con dolor discogénico mediante la clínica, radiología y discografía, debería permitirnos indicar y practicar técnicas como la INTRACEPT ${ }^{\circledR}$ e incluirlas en la cartera de servicios de las unidades del dolor (10).

\section{BIBLIOGRAFÍA}

1. Institute for Health Metrics and Evaluation (IHME). GBD Compare Data Visualization. Seattle, WA: IHME, University of Washington. 2020 [Accessed december 2020]. Disponible en: http://vizhub. healthdata.org/gbd-compare.

2. Encuesta Nacional de Salud de España 2017 [Internet]. Ministerio de Sanidad, Consumo y Bienestar Social. Disponible en: https://www.mscbs.gob.es/estadEstudios/estadisticas/encuestaNacional/encuestaNac2017/encuestaResDetall2017.htm

3. Manchikanti L, Benyamin RM, Singh V, Falco FJ, Hameed H, Derby R, et al. An update of the systematic appraisal of the 
accuracy and utility of lumbar discography in chronic low back pain. Pain Physician. 2013;16(2 Suppl): SE55-95.

4. Fischgrund JS, Rhyne A, Macadaeg K, Moore G, Kamrava E, Yeung $C$, et al. Long-term outcomes following intraosseous basivertebral nerve ablation for the treatment of chronic low back pain: 5-year treatment arm results from a prospective randomized double-blind sham-controlled multi-center study. Eur Spine J. 2020;29(8):1925-34. DOI: 10.1007/s00586020-06448-x.

5. Fischgrund JS, Rhyne A, Franke J, Sasso R, Kitchel S, Bae $\mathrm{H}$, et al. Intraosseous basivertebral nerve ablation for the treatment of chronic low back pain: a prospective randomized double-blind sham-controlled multi-center study. Eur Spine J. 2018;27:1146-56. DOI: 10.1007/s00586-0185496-1

6. Mu X, Wei J, A J, Li Z, Ou Y. The short-term efficacy and safety of artificial total disc replacement for selected patients with lumbar degenerative disc disease compared with anterior lumbar interbody fusion: A systematic review and meta-analysis. PLoS One. 2018;28:13:e0209660. DOI: 10.1371/journal. pone.0209660.

7. Ding F, Jia Z, Zhao Z, Xie L, Gao X, Ma D, et al. Total disc replacement versus fusion for lumbar degenerative disc disease: a systematic review of overlapping meta-analyses. Eur Spine J. 2017;26(3):806-15. DOl: 10.1007/s00586-016-4714-y.

8. Qaseem A, Wilt TJ, McLean RM, Forciea MA; Clinical Guidelines Committee of the American College of Physicians. Noninvasive Treatments for Acute, Subacute, and Chronic Low Back Pain: A Clinical Practice Guideline From the American College of Physicians. Ann Intern Med. 2017;166(7):51430. DOl: 10.7326/M16-2367.

9. Helm li S, Simopoulos TT, Stojanovic M, Abdi S, El Terany MA. Effectiveness of Thermal Annular Procedures in Treating Discogenic Low Back Pain. Pain Physician. 2017;20(6):447-70.

10. Fujii K, Yamazaki M, Kang JD, Risbud MV, Cho SK, Qureshi SA, Hecht AC, latridis JC. Discogenic Back Pain: Literature Review of Definition, Diagnosis, and Treatment. JBMR Plus. 2019;3(5):e10180. DOI: 10.1002/jbm4.10180. 